\title{
Potential common pathophysiological pathway of hypertension- mediated organ damage in hypertensive emergency
}

\author{
Naoki Nakagawa ${ }^{1} \cdot$ Naoyuki Hasebe $^{1}$
}

Received: 22 August 2020 / Revised: 28 August 2020 / Accepted: 28 August 2020 / Published online: 4 November 2020

(c) The Japanese Society of Hypertension 2020

Hypertension is an important public health issue because of its association with a number of serious diseases and adverse outcomes [1]. In particular, hypertensive emergency is not simply a condition in which the blood pressure (BP) level is abnormally high but can be associated with the presence of acute hypertension-mediated target organ damage [2]. Malignant hypertension (MHT), a form of hypertensive emergency, has traditionally been clinically diagnosed as the presence of severe BP in association with hypertensive retinopathy changes of hemorrhages and exudates with or without papilledema [3]. The pathophysiological hallmark of MHT is the presence of fibrinoid necrosis, which can involve multiple target organs, such as the brain, eyes, heart, and kidneys $[4,5]$. Thus, there is the view that the term "MHT" is obsolete and should be replaced by alternative terms, including "hypertensive emergency with multiorgan damage" [6]. A recent position document on hypertensive emergency from the European Society of Cardiology (ESC) Council on Hypertension reflects this view [7].

In the current issue of Hypertension Research, Mishima et al. report a detailed evaluation of concurrent target organ damage in patients with MHT at the time of clinical presentation [8]. The authors also report updated data on the short-term clinical course after 4 weeks of treatment and interorgan relationships in these patients based on matrix analysis. The main novelty of the study is perhaps the inclusion of a systematic evaluation of brain target organ damage, as characterized by posterior reversible encephalopathy syndrome (PRES) reflecting vasogenic edema involving the white matter of the parietal to occipital lobes found in all patients regardless of their neurological

Naoki Nakagawa

naka-nao@asahikawa-med.ac.jp

1 Division of Cardiology, Nephrology, Pulmonology and Neurology, Department of Internal Medicine, Asahikawa Medical University, Asahikawa, Japan symptoms. They found that target organ damage in the brain and serous retinal detachment (SRD) were most prevalent, i.e., in $60 \%$ of all examined patients. Second, the authors show the interaction of target organ damage using matrix analysis in which the complication rate of each symptom was demonstrated. Notably, the highest complication rates were found with PRES, SRD, and urinary protein $>1 \mathrm{~g} / \mathrm{g}$ creatinine. Among the 20 patients examined, 10 presented with all three symptoms, namely, PRES, SRD, and urinary protein $>1 \mathrm{~g} / \mathrm{g}$ creatinine, and 13 presented one of the symptoms; the remainder did not exhibit any of the three symptoms. These findings suggest that acute hypertensive target organ damage was concurrent across vital organs based on the dysfunction of arterioles as "strain vessels" that form large resistance gradients, which are important for the regulation of arterial pressure for organ perfusion [9].

A major increase in systemic and cerebral perfusion pressure, which occurs in MHT, disturbs autoregulation of the cerebral circulation, leading to the failure of autoregulatory cerebral vasoconstriction, with focal or generalized dilatation of small arteries and arterioles [6]. This results in cerebral blood volume overload, impairment of blood-brain barrier function and, in extreme cases, the development of brain edema. Importantly, these cerebral abnormalities occur in parallel with the systemic impairment of macrovascular and microvascular function, thereby limiting the ability to provide appropriate adjustment in the peripheral vascular tone. The first signs and symptoms of brain involvement are usually the sudden onset of headache, nausea, and vomiting, which may be followed by the development of different visual signs and visual field loss; in some cases, fluctuating neurological symptoms (restlessness; confusion; and, in extreme cases, seizures; and coma) may be present $[3,6]$.

The strain vessel hypothesis would also explain the close link between hypertensive retinopathy and cerebrocardiovascular diseases. The retinal artery is derived from the ophthalmic artery, which is the first branch emanating from the high-pressure internal carotid artery. Pressure in 
the central retinal artery, which can be measured by an ophthalmodynamometer, is approximately half of the systemic BP. Therefore, retinal circulation has hemodynamic characteristics similar to those of perforating arteries. Hypertensive retinopathy is a microvascular injury associated with increased BP that has long been an indicator of atherosclerosis. Indeed, the risk of incident stroke increases by $2-3$-fold in patients with retinopathy $[9,10]$. Hypertensive retinopathy is also associated with microalbuminuria, renal dysfunction, and left ventricular hypertrophy. Approximately two-thirds of patients with central retinal artery occlusion have high BP, and one-fourth have cardiovascular diseases $[9,10]$. Therefore, the strain vessel hypothesis may be one of the main theories explaining why hypertension and diabetes, unforeseen in the concept of evolution, preferentially affect vital organs such as the brain, eye, heart, and kidney.

MHT is a hypertensive emergency, and the ESC position document on the management of hypertensive emergencies recommends the use of intravenous BP-lowering medications in patients with acute presentations [7]. Nonetheless, the position document acknowledges that given the lack of randomized controlled trials on different treatment strategies, the recommendations are based on consensus from clinical experience. In the current report by Mishima et al. [8], the parallel improvement of PRES, SRD, and proteinuria after treatment is highly suggestive of a common pathophysiology among the three symptoms. Mechanistically, capillary leakage due to capillary hyperperfusion following the disruption of pressure regulation in arterioles is considered the common pathophysiology of these symptoms, though kidney damage in MHT includes irreversible glomerular sclerosis and reversible glomerular ischemia. Although accelerated malignant hypertension is regarded as urgent, it should be treated in accordance with emergencies. In many cases, treatment with oral drugs is possible. As many of these patients have a long history of hypertension, a rapid decrease in BP is associated with the risk of ischemia in important organs. The use of angiotensin-converting enzyme (ACE) inhibitors and angiotensin II receptor blockers (ARBs) in the acute phase must be commenced at low doses, as they could result in a rapid and dangerous fall in BP in some patients [2, 7]. One of the reasons for the profound BP drop is volume depletion, secondary to pressure natriuresis. To correct the circulating volume, intravenous saline infusion is recommended together with low-dose oral ACE inhibitors or ARBs. However, loop diuretics should be used if sodium/water retention is present $[2,7]$. BP should be reduced to no less than a diastolic pressure of $100-110 \mathrm{mmHg}$ during the first $24 \mathrm{~h}[2,7]$.
In conclusion, MHT is the most severe and one of the most challenging forms of hypertension, and it affects multiple organs. MHT is not yet an obsolete diagnosis, but evolution of the definition in the twenty-first century reflecting contemporary practice may be needed [7]. Nevertheless, the principles of early diagnosis, detailed systematic evaluation (including modern brain imaging), and effective treatment remain key factors for longer eventfree survival. Further data are clearly needed to assess the clinical significance of target organ damage and the interorgan relationship with patient survival and optimal longterm management.

\section{Compliance with ethical standards}

Conflict of interest The authors declare that they have no conflict of interest.

Publisher's note Springer Nature remains neutral with regard to jurisdictional claims in published maps and institutional affiliations.

\section{References}

1. Nakagawa N, Hasebe N. Impact of the 2017 American College of Cardiology/American Heart Association Blood Pressure Guidelines on the Next Blood Pressure Guidelines in Asia. Curr Hypertens Rep. 2019;21:2.

2. Umemura S, Arima H, Arima S, Asayama K, Dohi Y, Hirooka Y, et al. The Japanese Society of Hypertension Guidelines for the Management of Hypertension (JSH 2019). Hypertens Res. 2019; 42:1235-481.

3. Shantsila A, Lip GYH. Malignant hypertension revisited-does this still exist? Am J Hypertens. 2017;30:543-9.

4. Suzuki A, Nakagawa N, Maruyama K, Matsuki M, Hasebe N. Diffuse alveolar hemorrhaging with hypertensive emergency: a rare but important cause of hemoptysis. Intern Med. 2019;58:1511-6.

5. Nakagawa N, Duffield JS. Myofibroblasts in fibrotic kidneys. Curr Pathobiol Rep. 2013;1:189-98.

6. Cremer A, Amraoui F, Lip GY, Morales E, Rubin S, Segura J, et al. From malignant hypertension to hypertension-MOD: a modern definition for an old but still dangerous emergency. J Hum Hypertens. 2016;30:463-6.

7. van den Born BH, Lip GYH, Brguljan-Hitij J, Cremer A, Segura J, Morales E, et al. ESC Council on hypertension position document on the management of hypertensive emergencies. Eur Heart J Cardiovasc Pharmacother. 2019;5:37-46.

8. Mishima E, Funayama Y, Suzuki T, Mishima F, Nitta F, Toyohara $\mathrm{T}$, et al. Concurrent analogous organ damage in the brain, eyes, and kidneys in malignant hypertension: reversible encephalopathy, serous retinal detachment, and proteinuria. Hypertens Res. 2020; https://doi.org/10.1038/s41440-020-0521-2.

9. Ito S, Nagasawa T, Abe M, Mori T. Strain vessel hypothesis: a viewpoint for linkage of albuminuria and cerebro-cardiovascular risk. Hypertens Res. 2009;32:115-21.

10. Wong TY, Mitchell P. The eye in hypertension. Lancet. 2007;369: 425-35. 